\title{
CONGENITAL CORONARY ARTERIO-VENOUS ANEURYSM
}

\author{
BY
}

\author{
P. H. DAVISON, B. H. McCRACKEN, AND D. J. S. McILVEEN
}

From the General Hospital, Birmingham and Hill Top Hospital, Bromsgrove, Worcs.

Congenital arterio-venous aneurysms are not rare and it is, perhaps, surprising that they do not occur more frequently for the development of the vascular bed is extraordinarily complex. They are most commonly situated in the brain, limbs, or lungs, and the local and general disturbances of function that they produce are now well known. Involvement of the coronary circulation must be most unusual for only three instances have been reported in the English journals (Halpert, 1930; Paul et al., 1949). The following case is of interest because the arterio-venous aneurysm, which involved the circumflex branch of the left coronary artery, proved to be clinically indistinguishable from a patent ductus arteriosus, and the blood flow through it became so great that the patient died from congestive cardiac failure.

\section{Case Report}

Mrs. B.D., housewife of fifty-eight years, was admitted to the Birmingham General Hospital in February, 1954, with congestive cardiac failure. She had experienced bouts of palpitations for about ten years. In 1948 she was examined in a medical out-patient department when clinical and electrocardiographic examinations were normal and the symptoms were considered to be due to paroxysmal tachycardia. Subsequently she developed increasing dyspnœa on exertion, orthopnœa, and fatigue, and was admitted to another hospital in July, 1953. She was found to have congestive cardiac failure with auricular fibrillation. On repeated examinations a systolic murmur but no diastolic murmur was heard and the diagnosis made was rheumatic heart disease. She was re-admitted to the same hospital in November, 1953, again in cardiac failure, and during the course of this second admission, examinations by the same physician as before disclosed the development of a systolic and diastolic machinery type murmur in the pulmonary area. The cardiac failure responded to therapy but relapsed after she left hospital in spite of continued use of digitalis and mercurials and she was admitted to the General Hospital.

Previous Health. No rheumatic or other significant illness.

Family History. The patient was one of ten siblings of whom two died of " valvular heart disease " at the ages of eleven and fifty-four years respectively. Neither parent had known heart disease.

Clinical examination showed a rather thin woman in gross congestive failure with moderately severe dyspnœa, orthopnœea, and cyanosis. She was afebrile. The neck veins were distended and there was marked dependent œdema extending as high as the waist. There were râles at both lung bases and evidence of basal pleural effusions. The liver was enlarged $2 \cdot 5-5 \mathrm{~cm}$. below the right subcostal margin. The fingers were not clubbed. The pulse was completely irregular, of good volume and with a collapsing quality, and about 80 a minute. Blood pressure was $125 / 55$. The heart was enlarged with a heaving apical impulse in the anterior axillary line, five inches from the midsternum in the sixth left interspace. There was a visible and palpable systolic lift of the præcordium to the left of the sternum and a systolic thrill was palpable in the second and third left interspaces close to the sternum. At the apex the first sound was followed by a loud harsh blowing systolic murmur (grade 3/6) well conducted into the axilla. At the base the pulmonic element of the second sound was accentuated and a loud murmur which extended through systole and diastole was audible over a wide area, being maximal in the pulmonary area.

Investigations. Red blood cells, 5.4 million; hæmoglobin, 106 per cent (Haldane); white blood cells, 8800; E.S.R., $1 \mathrm{~mm}$. per hour (Westergren); Wassermann, negative; blood cultures, negative; serum chemistry -urea, $51 \mathrm{mg}$ per cent; sodium, 130 m.eq./litre; potassium, 5.4 m.eq./litre; chloride, 97 m.eq./litre. X-ray films of chest showed generalized enlargement of the heart with pulmonary congestion and basal pleural effusions. The patient was too ill to fluoroscope. Electrocardiograms showed auricular fibrillation, right axis deviation, and neither right nor left ventricular preponderance. (The cardiogram in 1948 showed normal sinus rhythm and normal ventricular axis in standard limb leads.) 
Hospital Course. The clinical diagnosis after admission was congestive cardiac failure due probably to patent ductus arteriosus. Treatment was aimed at improving the patient's condition enough to justify transferring her to the cardiac surgery unit at Hill Top Hospital. Digitalis, salt restriction, and oedema drainage by Southey's tubes did in fact produce a considerable improvement in the degree of cardiac failure, but two weeks after admission she developed a severe mental disorder with some classical features of a chronic schizophrenic reaction, negativism with resistance, at times violent, and periods of echopraxia and echolalia. No explanation for this occurrence was found in her serum chemistry, which was essentially the same as on admission, or in her previous mental history which had been impeccable. Periods of violence caused a relapse of cardiac failure and the outlook seemed serious enough to justify a trial of electroconvulsive therapy. A single treatment was followed after a few days by return to a fairly normal mentality. Her cardiac failure subsequently was relieved enough for her to be transferred to Hill Top Hospital seven weeks after admission. Cardiac catheterization was performed five days after her transfer in order to confirm the diagnosis of patent ductus before operation was undertaken.

Cardiac Catheterization. This was performed in the usual manner. Blood gas was analysed by the method of Van Slyke and Neill. Expired air was collected in a Tissot spirometer and analysed in a Sleigh general apparatus. The chambers of the right heart and the pulmonary artery were dilated. Attempts to enter a patent ductus were unsuccessful despite good catheter control. The findings, which are summarized in Table I, demonstrated a large left-to-right shunt into the right atrium, moderate pulmonary hypertension and high-output congestive heart failure.

\section{TABLE I}

\section{Cardiac Catheterization}

Mrs. B. D., 58 years, 6/3/54. Height, 61 in.; weight, 119 lb.; surface area, 1.51 sq. m.

Pressures recorded in $\mathrm{mm}$. $\mathrm{Hg}$ and measured from the sternal angle. Blood oxygen content expressed in volumes per $100 \mathrm{ml}$.

\begin{tabular}{|c|c|c|c|c|c|c|c|c|}
\hline \multirow{2}{*}{\multicolumn{5}{|c|}{ Site }} & \multicolumn{2}{|c|}{ Pressures } & \multicolumn{2}{|c|}{ Blood samples } \\
\hline & & & & & $\mathrm{s} / \mathrm{d}$ & mean & $\mathrm{O}_{2}$ content & $\%$ saturation \\
\hline $\begin{array}{l}\text { Left pulmonary artery } \\
\text { Right pulmonary artery } \\
\text { Main pulmonary artery } \\
\text { High right ventricle } \\
\text { Low right atrium ... } \\
\text { High right atrium .. } \\
\text { Left brachial artery }\end{array}$ & $\begin{array}{l}. \\
\therefore \\
. \\
\therefore \\
\therefore\end{array}$ & $\begin{array}{l}. \\
\ldots \\
\ldots \\
\ldots \\
\ldots\end{array}$ & $\begin{array}{l}. \\
\ldots \\
. \\
\ldots \\
\ldots \\
\ldots\end{array}$ & $\begin{array}{l}. \\
\therefore \\
. \\
. \\
\cdots \\
\cdots\end{array}$ & $\begin{array}{l}\overline{-} \\
\overline{75 / 25} \\
76 / 14 \\
\overline{-} \\
\overline{140 / 60}\end{array}$ & $\begin{array}{l}46 \\
43 \\
45 \\
14 \\
14 \\
88\end{array}$ & $\begin{array}{l}14 \cdot 31 \\
14 \cdot 04 \\
14 \cdot 13 \\
14 \cdot 42 \\
13 \cdot 31 \\
10 \cdot 73 \\
16 \cdot 87\end{array}$ & $\begin{array}{l}73 \\
71 \cdot 5 \\
72 \\
73 \cdot 5 \\
68 \\
54 \cdot 5 \\
86\end{array}$ \\
\hline
\end{tabular}

Oxygen capacity of blood: $19.66 \mathrm{vol} . / 100 \mathrm{ml}$.

Resting oxygen consumption: $192 \mathrm{ml} . / \mathrm{min}$.

Resting cardiac output: $7 \cdot 1$ 1./min.; $4 \cdot 71 . / \mathrm{min} . / \mathrm{sq} . \mathrm{m}$.

Effective systemic blood flow: $3 \cdot 1$ 1./min.; $2 \cdot 11$./min./sq. m.

Left-to-right shunt: $3.91 . / \mathrm{min}$., equal to 56 per cent of total cardiac output.

The arterio-venous oxygen difference of the effective systemic blood flow was calculated from the high right atrial blood sample which probably approximates fairly closely to mixed venous blood.

In view of the unexpected results the operation was not undertaken. The patient's condition deteriorated steadily and she died four weeks after admission.

Necropsy. Apart from the heart the findings were not remarkable and consistent with severe, prolonged congestive heart failure. The heart weighed $490 \mathrm{~g}$. and all chambers were dilated and hypertrophied. The ventricular and auricular septa were both intact and the foramen ovale closed. The mitral ring was dilated and the cusps a little thickened. The tricuspid ring was dilated and there was a plaque of thickened endocardium on the atrial aspect of the anterior cusp. The pulmonary valves were competent and the pulmonary artery dilated and thickened. The aortic valves were competent and normal apart from thickening of the left coronary cusp. The aorta showed minimal atheroma and the ligamentum arteriosum was firmly occluded. The right coronary artery was normal in origin, size and distribution and was free from atheroma. The left coronary aortic sinus was dilated, and the mouth of the left coronary artery so large that it was difficult to say where sinus ended and artery began. The left coronary artery was dilated and funnel-shaped. Its anterior descending branch arose normally, followed an orthodox course and was free from atheroma. The circumflex branch was thin-walled and grossly dilated throughout its entire length with an average diameter of $15 \mathrm{~mm}$. (Fig. 1). It was greatly elongated and the dilated loops, coiled in serpentine fashion, were firmly adherent to each other and to the inferior aspect of the left atrial appendage 


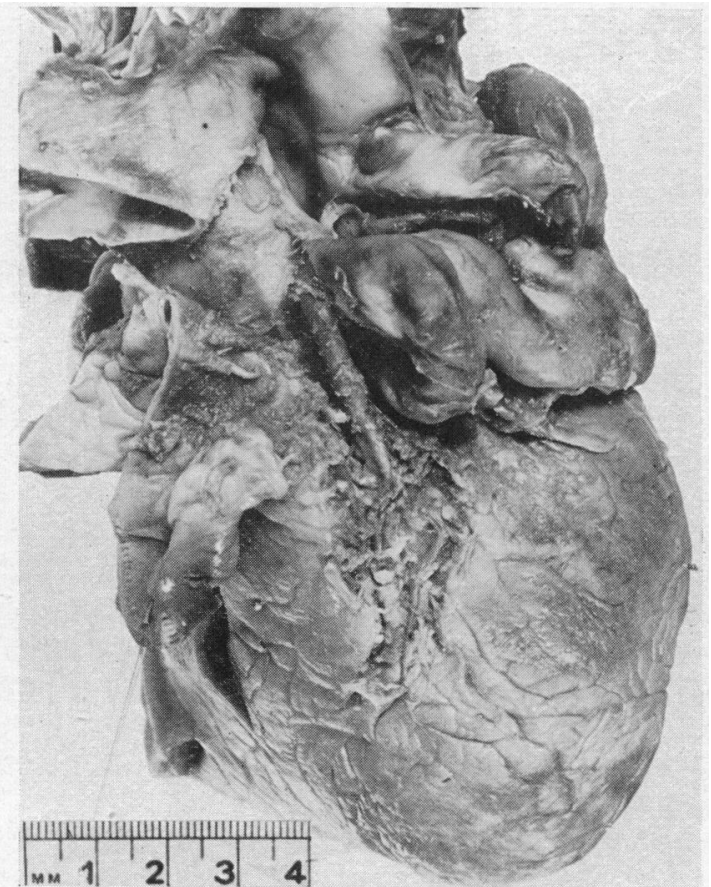

Fig.1.-The heart from the antero-lateral aspect. The main pulmonary artery has been divided to expose the first part of the aorta, the left coronary artery and its branches. The dilated circumflex branch passes backwards beneath the left atrial appendage.

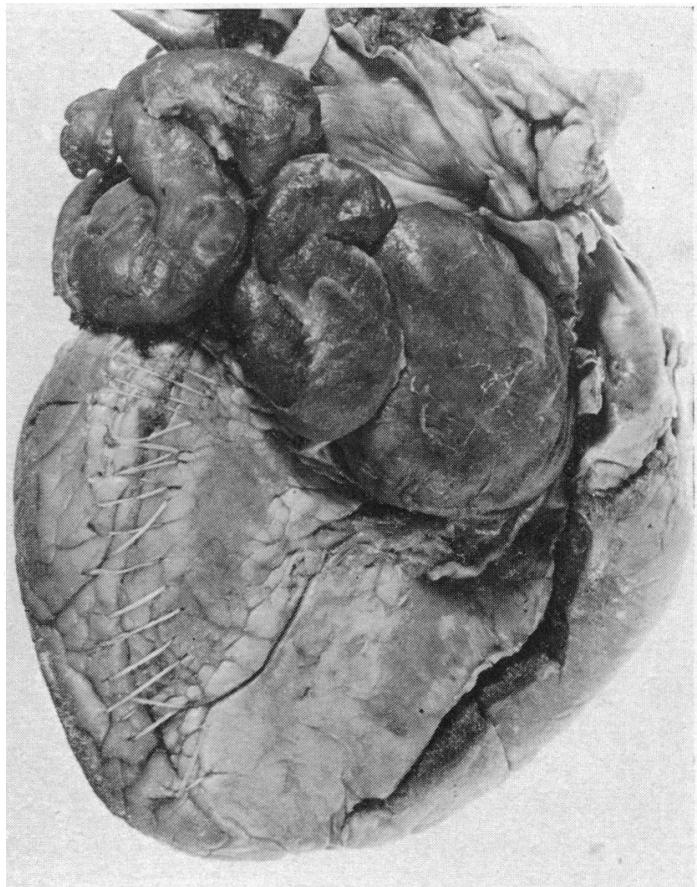

FIG. 2.-The heart from the postero-inferior aspect. The dilated circumflex artery is shown passing directly into the aneurysmal coronary sinus.

as they coursed backwards in the atrio-ventricular groove, overlying the great cardiac vein which was normal in size and position. The origins of the smaller branches of this dilated vessel, which normally supply the lateral and inferior aspects of the left ventricle, could not be demonstrated without destroying the specimen, but section through the lateral wall of the left ventricle showed branches of normal size. This dilated circumflex artery passed directly into the coronary sinus (Fig. 2). The latter was aneurysmally dilated and formed a thin-walled bag of blood $10 \mathrm{~cm}$. in diameter on the postero-inferior aspect of the heart. The venous drainage into the coronary sinus appeared normal. Its anterior wall projected into the right atrium and the medial wall of the atrium in this area was so thin that it was accidentally torn at one point during dissection of the heart. The mouth of the coronary sinus was oval in shape and measured $8 \times 5 \mathrm{~mm}$. Its lips were thickened and rigid, projecting into the right atrium like the crater of a volcano. The jet of blood from this orifice impinging upon the anterior leaflet of the tricuspid valve was presumably responsible for the plaque of thickened endocardium observed there. Histological sections of the heart muscle taken from the lateral wall of the left ventricle showed no evidence of myocardial fibrosis; the capillaries and larger vessels were well filled with blood.

\section{Discussion}

The discovery of an arterio-venous aneurysmal communication between the circumflex branch of the left coronary artery and the coronary sinus at necropsy explained the anomalous clinical and physiological findings during life. In the absence of trauma and infective or degenerative changes in the vessel it is reasonable to conclude that the arterio-venous fistula was congenital in origin. Congenital arterio-venous aneurysms are known to increase gradually in size over the course of years and this necessitates a progressive rise in the cardiac output in order to maintain an adequate orthodox circulation in the presence of an increasing shunt. This hyperkinetic state may lead, ultimately, to congestive cardiac failure. In this patient the absence of any symptoms of coronary 
insufficiency, the histological normality of the myocardium and the high-output congestive failure demonstrated by cardiac catheterization all indicate that death was directly attributable to the size of the shunt. This is not surprising for the aneurysm was taking approximately one-half of the total resting cardiac output.

In the previous reports of this anomaly (Halpert, 1930; Paul et al., 1949) the right coronary artery was involved in two cases and probably a branch of the left coronary artery in the third. In Halpert's patient the condition gave rise to no symptoms or signs during life and was a necropsy finding in a man of 54 years who died from carcinoma of the stomach. In the first case described by Paul and his colleagues an arterio-venous aneurysm of the right coronary artery was discovered at exploratory thoracotomy in a boy of nine years with a continuous murmur in the fourth right interspace who had no disability and only slight cardiac enlargement. A full report is not available in their second case but the condition was again discovered at thoracotomy in a boy of 16 years who was thought to have a patent ductus arteriosus. At operation the murmur was found to arise from an abnormal bulge on the lateral wall of the left ventricle and this was attributed to an arteriovenous aneurysm of the coronary circulation.

A correct ante-mortem diagnosis was not made in the case reported here although this would have been possible from the data available. The clinical diagnosis of patency of the ductus arteriosus was firmy excluded by the findings at cardiac catheterization. The clinical and catheter findings were consistent with rupture of the right aortic sinus into the right atrium. In the elderly this condition is usually a complication of syphilitic or mycotic aneurysm of the aortic sinus. The absence of these infections and the competence of the aortic valve were against this diagnosis.

It would be difficult to make a confident diagnosis of coronary arterio-venous fistula during life without resort to exploratory thoracotomy. If the condition is suspected then direct sampling of coronary venous blood with a cardiac catheter should be attempted although the large venous outflow would make its introduction into the coronary sinus difficult. Normal coronary venous blood contains little oxygen and an oxygen saturation exceeding 30 per cent of capacity may be taken as evidence of an arterial shunt into the coronary circulation. This can arise from a coronary arteriovenous aneurysm or from anomalous pulmonary veins draining into the right atrium via a persistent left superior vena cava and the coronary sinus. General signs of systemic arterio-venous fistula should serve to distinguish the former condition. Retrograde aortic angiography might prove a simpler method of demonstrating the abnormal vessels.

Unfortunately such diagnostic perfectionism would bring no therapeutic reward for ligation of the abnormal vessel would carry a grave risk of death from myocardial ischæmia.

\section{Summary}

A 58-year-old woman presented with clinical features consistent with congestive cardiac failure secondary to a large patent ductus arteriosus. Cardiac catheterization demonstrated an arterial shunt into the right atrium. A congenital arterio-venous communication between the circumflex branch of the left coronary artery and the coronary sinus was discovered at necropsy.

We wish to thank Miss S. Segel for technical assistance and Mr. W. Dee for the photographs.

\section{References}

Halpert, B. (1930). Heart, 15, 129.

Paul, O., Sweet, R. H., and White, P. D. (1949). Amer. Heart J., 37, 441. 